\section{The limits of reductionism}

SIR-The differences between myself and my friend Steven Weinberg ${ }^{\prime}$ might seem merely to be a semantic argument. Actually they touch upon some of the deepest questions of the Universe and our hopes to solve them. The ambiguous application of the term 'reduction' to a number of different scientific approaches has been the cause of much confusion.

One of the most successful methods of science is that of analysis. It means taking a phenomenon or process of nature and trying to dissect it into its components, and then dissecting again each of these components into its components until the point is reached where one cannot go any further. For instance, after having studied the function of the liver as a whole, I learn a great deal more by studying what goes on in individual liver cells, and finally by determining which molecules play which role in the different organelles of the liver cell etc.

This analytic process is sometimes referred to as constitutive reductionism because it consists of the reduction of the studied object into its most basic constituents. No scientist objects to this form of reduction, but it would seem less confusing if such analysis were not referred to as reduction.

Another phenomenon that has been called reduction is theory reduction, defined by $\mathrm{Nagel}^{2}$ as "the phenomenon of a relatively autonomous theory becoming absorbed by, or reduced to, some other more inclusive theory". The classical example is the reduction of thermodynamics to statistical mechanics. The number of theories that can thus be reduced seems quite limited, and it has not been possible to reduce without residue any biological theory to a physical theory? Weinberg agrees with this evaluation of theory reduction.

Where the argument begins is when one asks whether the knowledge of the basic constituents of an analysis would permit one to build up an understanding of the total system. We know that an inventory of all the molecules in the liver is not sufficient to reconstruct a description of the function of the entire liver. Without a knowledge of mitochondria and other cellular organelles and structures (membranes), without understanding blood circulation and the structure of capillaries, without knowing what the normal input and output of the liver is, and without a knowledge of many other aspects of the liver and the body as a whole, it would be utterly futile to try to arrive at a correct picture of liver function.

The view that the mere knowledge of its ultimate components would be sufficient to explain a complex system has been referred to by me as explanatory reduc- tionism ${ }^{4}$. It has been rejected by many distinguished physicists, including Niels Bohr, and Weinberg agrees with them. Even if you knew everything about every molecule in a glass of water, he says, "how would you ever recognize the properties that interest you about water, properties like vorticity, turbulence, entropy and temperature?"'. He realizes that "as one goes to the higher and higher levels of organization, new concepts emerge that are needed to understand the behaviour at that level" . This acceptance of emergence is quite a departure from the view formerly held by many philosophers and physicists.

Then where do Weinberg and I disagree? Some philosophers have proposed that man in his search for truth deals with three worlds: the microworld of subatomic particles, the middle world from the atom to our Solar System, and the megaworld of the infinite cosmos. Our system of cognition is adapted to deal with the tangible middle world. It is my contention that advances in our understanding of the microworld of subatomic particles are not going to make any explanatory contributions to our understanding of the middle world.

When Weinberg disowned explanatory reductionism, he implicitly agreed with this view. Yet a number of exuberant statements on the last two pages of his article $^{\prime}$ suggest that he expects from the Superconducting Super Collider (SSC) more than a slight advance in our understanding of the subatomic world. He says that "the arrows of explanation point down to a common source" and that in particle physics "we are at a level which is in fact in absolute terms quite deep, perhaps close to the final source", and that the particle physicist finds a "simplicity, a beauty, .... in the rules that govern matter that mirrors something that is built into the logical structure of the Universe at a very deep level".

I am willing to grant Weinberg that all this might indeed be true as far as the ultimate structure of matter is concerned. I am not a physicist but I would even be willing to accept the claim that some of the findings made in subatomic physics might help to explain certain phenomena of the megaworld. However, Weinberg's stress on the importance of this research implies a hope that it would contribute not only to our understanding of the subatomic world, but also to that of the middle world. I am not so euphoric. Even if the $\mathrm{SSC}$ would lead to the discovery of the Higgs boson or some other equivalent finding, my disbelief in explanatory reductionism leads me to doubt that such a discovery would make any contribution whatsoever to our understanding of the middle world. But it is the middle world that poses all the problems man will have to solve if he is to survive.

I conclude therefore that it is more than doubtful whether the expenditure of 4.4 billion dollars (or more) for the discovery of the Higgs boson can be justified. To be sure, the construction of the SSC is heavily favoured by certain industries and by those politicians who hope that the project will be placed in their state, but whether science as a whole and the United States will derive benefits from this project remains a justified question.

Museum of Comparative Zoology,

ERNST MAYR

Harvard University,

Cambridge, Massachusetts 02138, USA

1. Weinberg, S. Nature 330, 433-437 (1987)

Nagel, E. The Structure of Science 336 (Harcourt Brace. and World, New York, 1961)

3. Ayala, F.J. and Dobzhansky. Th. (eds) Studies in the Phil. osophy of Biology (University of California Press. Los Angeles, 1974)

4. Mayr. E. The Growth of Biological Thought 60 (Harvard University Press. Cambridge. Massachusetts, 1982).

WEINBERG REPLIES - I can't help but feel that if I could only express myself a little more clearly, Ernst Mayr and I would see that there's really no disagreement between us. So here is one more attempt.

Perhaps the main point that needs clarification is that when I speak of theories as explaining natural phenomena, I am taking for granted a kind of transitivity. If a large number of experimental facts $a, b, c$, . are explained by a set of theories $\mathrm{X}, \mathrm{Y}$,

., and then these theories are in turn explained by a more satisfying theory $\mathrm{Z}$. then I would say that the facts a,b,c,

are explained by theory $Z$ even though we already understood them in terms of the previous theories $\mathrm{X}, \mathrm{Y}, \ldots$. This may $\mathrm{I}$ suppose be regarded as just a matter of definition; for me the important thing is that in explaining theories $\mathrm{X}, \mathrm{Y}, \ldots$. . the new theory $Z$ gives us a sense that we better understand why our world (including facts $a, b, c, \ldots$. ) is the way it is, and that this is one factor that (among other factors) ought to be taken into account in making decisions about the support of scientific research.

To take one example within physics itself: Newton's theories of gravitation and of motion explained a vast number of facts about our Solar System, and about the motion of projectiles and the oceans here on Earth. However, these theories involved several apparently arbitrary elements: the inverse square law, the equality of gravitational and inertial mass, and so on. Also, as realized later, they were in conflict with special relativity. Newton's theories were then explained as an approximate consequence of a more satisfying theory, Einstein's general theory of relativity. In a narrow sense, general relativity contributed very little to our understanding of the Solar System or the tides. We already knew enough to cal- 
culate planetary orbits with great precision, and general relativity did not help us with the major puzzles still outstanding (long-term stability, tidal dissipation), only with one tiny anomaly in the orbit of Mercury. Nevertheless, the development of general relativity was important, at least in part, because it explained the theories of Newton that had earlier explained so much else.

Of course. general relativity is important also because it predicts new phenomena. such as black holes and gravitational lenses. This is our historical experience; a theory that provides a more satisfying explanation of what we already knew is likely also to predict things of which we had not yet dreamed.

I suspect that Ernst Mayr would not really disagree with these remarks about general relativity. But in his response to my article he marks out a 'middle world', of scales from the atom to the Solar System, and expresses the doubt that discoveries in elementary particle physics, such as those expected at the SSC accelerator, "would make any contribution whatsoever to our understanding of the middle world". I suppose that he must mean to restrict this remark to future discoveries after all, everyone knows that the discovery of the electron and the atomic nucleus and the quantum mechanical description of their interaction made an enormous contribution to our understanding of matter at the scale of ordinary life. But our quantum theories of electrons and atomic nuclei are clearly not complete - they contain a large number of seemingly arbitrary elements, for instance the fact that the electron is some 2,000 times lighter than the particles in the atomic nucleus. Also, they leave out gravitation.

We are trying to develop a more satisfying theory that explains all these mysteries, a task for which we need new instruments like the SSC. It may be that such a theory would not make life any easier for the fluid dynamicist or the evolutionary biologist, just as general relativity did not help very much in the actual work of celestial mechanics and planetary physics. Yet I think it fair to say that, like general relativity, the sort of theory that we are aiming at in particle physics would, if only by explaining our previous theories, contribute fundamentally to our understanding of the 'middle world'. And if history is any guide, it would predict exciting new phenomena as well.

In the end, we would not be very happy with an understanding of nature that rests on any fundamental distinction between the microworld, middle world and megaworld. We live in one world, and in trying to understand it we discover chains of explanation that cannot be followed farther without looking deeper into the physics of elementary particles. Following these chains down to their roots is not the only kind of science that is important, but how can anyone doubt that it is important?

Theory Group,

Physics Department,

University of Texas at Austin,

Austin, Texas 78712, USA

\section{Replacing tenure}

SIR-D.A.W. Grant and Bruce Charlton in their letters of protest (Nature 328, 754; 1987) about the plight of UK science are both right and both wrong. They are correct to complain about the lack of effective leadership and about the undue acquiescence of bodies such as the Advisory Board for the Research Councils (ABRC) in time- and resource-wasting schemes to give a spurious sense of becoming more efficient by centralizing resources. The shambles created in London medical schools by such an approach should be enough to give pause for thought, before embarking on a much larger exercise based on the same false premise that such reorganizations save money. They do not, and, worse, they are very disruptive. Grant and Charlton are wrong in castigating Nature for editorial comments on our plight; we should have listened harder and replied more effectively to both government criticism and Nature's prodding. To take a specific point of Grant's, whether the abolition of tenure is good or bad depends on what takes its place. At present, the major advantage of tenure is a practical one: it allows recruitment of staff at lower salaries than would otherwise be the case; even so, we are still losing out to industry and overseas where tenure is much less common. Competitive salaries for (say) rolling 5-year contracts would make us a lot more able to retain or recruit outstanding staff. The main disadvantage of tenure, which is continually hung round our necks and to which we reply so ineffectively, is that it protects the right to idleness (rather than the academic freedom about which we are now hearing such a lot).

The current proposals on tenure will give us the worst of all worlds - new young staff without tenure and existing staff reluctant to move because they will lose tenure; if they do move, it will probably be out of the universities altogether. If the government were to realize the true economic cost of abolishing tenure, it might be persuaded to make more sensible proposals. Tenure should either be abolished for all, with government providing funds to run an economically competitive scheme, or it should be retained (for largely economic reasons) but the universities should be provided with the means and resources to redeploy ineffective staff. Either approach would give us the flexibility to create careers that would be once again attractive to bright young academics. Our young colleagues' morale is depressed not only by the difficulty of attracting research grants but also by the apparent inability of universities to deal with chronic institutional stasis of life-threatening proportions. To be sure, more money would help, but we are more likely to get it if we show a determined effort to set our own houses in order.

\section{University of Southampton, \\ Department of Physiology \& \\ Pharmacology, \\ Medical \& Biological Sciences Building, \\ Bassett Crescent East,}

Southampton SO9 3TU, UK

\section{Creationists now}

SIR-With reference to Reginald T. Chelvam's letter (Nature 331, 10:1988), our present knowledge is the compound of millennia of accumulation. As prehistoric man pondered on his relationship with the Universe, he encountered the obstacle of the past. The origins of life and the physical world were shrouded in mystery, which was surmounted only by the creation of a 'superhuman' crutch to insecurity. Belief in these various deities has been passed down through the ages. This faith has become so entrenched in man's teachings that many find it hard to accept that the evolutionary theory of the origin of life, and in a wider context matter, has its merits. The more insecure individuals would obviously prefer to cling to the older theory. They are then the centre of the Universe, and life has a purpose and is not the product of aeons of random occurrences.

How can Chelvam equate the narrowminded creationists of today with the 'default', if you will, creationist founders of our science, who had not even conceived of alternative explanations of origins? Darwinism was never specifically proposed to negate the existence of gods - evolution merely obviated their involvement in the creation of life. There are many (confused?) scientists who believe in evolution and retain a belief in an omniscient, omnipotent being.

We have a long way to go before we understand the Universe, if we ever do. Evolutionary and other theories may be a step on that path, though the teaching of creationism to the next generation as the one and true road will corrupt the attitudes of our future philosophers and scientists. The various theories on the origins of life should be presented to children. They will make up their own minds one day, why not sooner rather than later? Or is it their greater mental freedom that the forces in the Bible Belt fear?

ANDREW W.P. ROBERTS Bracknell RG12 4XZ, UK 